\title{
On the Boundary Layer in Singularly Perturbed Problem with An Initial Jump
}

\author{
Assiya Zhumanazarova \\ Department of Computer Engineering, Gachon University, Gyeonggi-do 461-701 \\ Seongnam, Korea \\ Young Im Cho \\ Department of Computer Engineering, Gachon University, Gyeonggi-do 461-701 \\ Seongnam, Korea \\ E-mail: assiya_mukhamaddin@mail.ru,yicho@gachon.ac.kr \\ www.gachon.ac.kr
}

\begin{abstract}
In this study, we consider the foundations of the boundary layer theory for studying the boundary layer part of the asymptotics of a singularly perturbed integro-differential boundary value problem with an initial jump. A part of the boundary layer is defined as an expansion in powers of a small parameter, and the coefficients of this expansion are solutions to differential initial value problems. The purpose of this study is to describe in detail the properties of the boundary layer part of the asymptotics based on the principles of boundary layer theory.
\end{abstract}

Keywords: boundary layer, singular perturbation, initial jump, exponential estimates.

\section{Introduction}

The boundary layer theory studied by Prandtl has been applied to characterize the problems of external flow around a body at high flow degree of velocity or low viscosity. Various fundamental problems of mechanics and physics can be formulated using a significantly simplified model using the approximate theory of the boundary layer. The results of analytical solutions to these problems are provided with a high degree of accuracy. The practical significance of the study of boundary layers lies in the fact that they take place in many important processes, where the effect of fluid viscosity is significant in the neighborhood of the boundary surface. One of the specific directions of the boundary layer theory is the friction layer in aerodynamics, where it is characterized by a strong gradient of flow velocity. To the theoretical study of the flow of low-viscosity fluids, the entire field can be divided into two parts: the region of a thin boundary layer near the wall, where friction forces must be considered, and the region outside the boundary layer, where the friction forces can be ignored due to their smallness. ${ }^{1}$ The approximate character of the considered theory is that it is based on the assumption of a small thickness of the boundary layer.

The boundary layer theory can be considered from a physical and mathematical point of view. The theory of differential equations containing small parameters at the highest derivatives is important in mathematical modeling of physical processes. In the theory of singularly perturbed boundary value problems, a boundary layer phenomenon is observed when the solution to the perturbed problem differs significantly from the solution of a degenerate problem for arbitrarily small parameters. The behavior of the solution of a singularly perturbed system in the boundary layer is described by exponentially decaying functions. ${ }^{2}$ The system describing a viscous fluid is an example of a singularly perturbed system. In hydrodynamics, the 
equation of an ideal fluid even at low viscosity is not suitable for describing a process near the boundary, called the boundary layer. ${ }^{2}$ In perturbation theory the method of matched asymptotic expansions was developed to find an approximate solution by constructing different asymptotic solutions inside and outside the boundary layer of rapid changes and then, matching them together to obtain a unique approximate solution. ${ }^{3}$

In present section, we considered the basic concepts of the boundary layer theory to find association between their practical and theoretical importance. In the following section, we consider a singularly perturbed problem with an initial jump where the foundations of the theory of boundary layers are applied. ${ }^{4}$

The remainder of this paper is organized as follows. Section 2 is devoted to describe the main points based on the results of previous studies on singularly perturbed boundary value problem with an initial jump and to the formation of boundary layer part of asymptotics of the solution. Finally, Section 3 provides some concluding remarks.

\section{The Boundary Layer Part of Asymptotics}

Consider a singularly perturbed integro-differential boundary value problem with an initial jump. ${ }^{5,6}$ In these studies, singularly perturbed and unperturbed problems, as well as the convergence of solutions using asymptotic methods are investigated. Since the presence of an integral term in the original equation significantly affects the solution, the phenomenon of an initial jump was observed in the problem at the left point of the considered segment. To form a uniform asymptotic expansion considering the asymptotic properties of solutions, the method of boundary functions was used. Thus, an algorithm was formulated for constructing asymptotic expansion of solution, which was characterized by a rapid change within the boundary layer. The asymptotics was sought as the sum of two parts - regular and boundary. A uniform asymptotic expansion of the solution is obtained by solving a sequence of simpler problems for finding the composite parts of the asymptotics. That is, a uniform asymptotic expansion has the form ${ }^{6}$

$$
y(t, \varepsilon)=\sum_{m=0}^{n} \varepsilon^{m} y_{m}(t)+\sum_{m=0}^{n+2} \varepsilon^{m} \rho_{m}(\tau)+r_{n}(t, \varepsilon) .
$$

Here, $y_{m}(t)$ and $\rho_{m}(\tau)$ denote the regualar and boundary layer terms, respectively, and $r_{n}(t, \varepsilon)$ denotes the remainder term of the asymptotics.

Consider the differential initial value problems defined for finding the boundary layer terms

$$
\begin{aligned}
& \dddot{\rho}_{m}(\tau)+A(0) \ddot{\rho}_{m}(\tau)+B(0) \dot{\rho}_{m}(\tau)=\psi_{m}(\tau), m \geq 0, \\
& \psi_{0}(\tau) \equiv 0, \\
& \rho_{0}(0)=-\Delta_{0}, \dot{\rho}_{0}(0)=0, \ddot{\rho}_{0}(0)=B(0) \Delta_{0}, \\
& \rho_{1}(0)=-\Delta_{1}, \dot{\rho}_{1}(0)=\beta-y_{0}^{\prime}(0), \\
& \ddot{\rho}_{1}(0)=-A(0)\left(\beta-y_{0}^{\prime}(0)\right)+B(0) \Delta_{1}-\int_{0}^{\infty} \psi_{1}(p) d p, \\
& \rho_{m}(0)=-\Delta_{m}, \dot{\rho}_{m}(0)=-y_{m-1}^{\prime}(0), \\
& \ddot{\rho}_{m}(0)=A(0) y_{m-1}^{\prime}(0)+B(0) \Delta_{m}-\int_{0}^{\infty} \psi_{m}(p) d p, m \geq 2 .
\end{aligned}
$$

By direct calculations, we obtain the solution for the 0 -th order approximation in the form

$$
\begin{aligned}
\rho_{0}(\tau)= & -\frac{B(0) \Delta_{0}}{\mu_{2}(0)-\mu_{1}(0)}\left(\frac{1}{\mu_{1}(0)} \mathrm{e}^{\mu_{1}(0) \tau}-\frac{1}{\mu_{2}(0)} \mathrm{e}^{\mu_{2}(0) \tau}\right)+ \\
& +\frac{\left(B(0)-\mu_{1}(0) \mu_{2}(0)\right) \Delta_{0}}{\mu_{1}(0) \mu_{2}(0)} .
\end{aligned}
$$

It follows immediately from the solution that

$$
\left|\rho_{0}^{(j)}(\tau)\right| \leq \mathrm{M} e^{-\delta \tau}, j=\overline{0,2, \tau} \geq 0 .
$$

Here, M, $\delta$ are positive constants.

To find the solutions to problems (2), (3), $m \geq 1$ that is, for the non-homogeneous differential equations (2), $m \geq 1$ we have used the Lagrange's method of variation of constants. After finding the solutions $\rho_{m}(\tau), m \geq 1$ and their derivatives of first and second order, we have obtained their exponential estimates. Using the method of mathematical induction, that is, supposing that the estimates are valid up to the $m$-1-th order approximation, we prove them for the $m$-th order approximation.

The boundary layer phenomenon can occur when solving a boundary value problem for an integrodifferential equation with a small parameter. The degenerate equation obtained by equating a small parameter has an initial jump in the integral term, as well as in the boundary conditions, modification is required, in consequence of which a boundary layer occurs. 


\section{Conclusion}

In this study, we considered the main concepts of the boundary layer theory for studying a singularly perturbed integro-differential boundary value problem with an initial jump. To describe their properties, the boundary layer part of the asymptotic expansion of the solution was considered. The study revealed that the terms of the boundary layer part of the asymptotics are characterized by exponentially decreasing functions. In conclusion, having studied the foundations of the theory of the boundary layer, it was possible to characterize the properties of the boundary layer part of the asymptotic expansion of the solution. Further work is required to expand this study, including fully proving boundary layer term estimates and providing detailed solutions to nonhomogeneous differential equations.

\section{Acknowledgements}

This research was supported the National Research Foundation (NRF) - 2018R1D1A1A09084151.

\section{References}

1. H. Schlichting, Boundary-Layer Theory, Nauka, Moscow, 1974.

2. A.B. Vasilyeva and V.F. Butuzov, Asymptotic Expansion of Solutions of Singularly Perturbed Equations, Nauka, Moscow, 1973.

3. E.J. Hinch, Perturbation Methods, Cambridge University Press, Cambridge, 1991.

4. K.A. Kasymov, Singularly Perturbed Boundary Value Problems with Initial Jumps, Sanat, Almaty, 1997.

5. A. Zhumanazarova and Y.I. Cho, Asymptotic convergence of the solution of a singularly perturbed integrodifferential boundary value problem, J. Mathematics, 8(213), 2020, pp. 1-17.

6. A. Zhumanazarova and Y.I. Cho, Uniform Approximation to the Solution of a Singularly Perturbed Boundary Value Problem with an Initial Jump, J. Mathematics, 8(2216), 2020, pp. 1-14. 